\title{
On the Lattice of Intervals and Rough Sets
}

\author{
Adam Grabowski \\ Institute of Mathematics \\ University of Białystok \\ Akademicka 2, 15-267 Białystok \\ Poland
}

\author{
Magdalena Jastrzębska \\ Institute of Mathematics \\ University of Białystok \\ Akademicka 2, 15-267 Białystok \\ Poland
}

Summary. Rough sets, developed by Pawlak [6], are an important tool to describe a situation of incomplete or partially unknown information. One of the algebraic models deals with the pair of the upper and the lower approximation. Although usually the tolerance or the equivalence relation is taken into account when considering a rough set, here we rather concentrate on the model with the pair of two definable sets, hence we are close to the notion of an interval set. In this article, the lattices of rough sets and intervals are formalized. This paper, being essentially the continuation of [3], is also a step towards the formalization of the algebraic theory of rough sets, as in [4] or [9].

MML identifier: INTERVA1, version: $7.11 .04 \quad 4.130 .1076$

The articles [2], [1], [10], [7], [3], [5], and [8] provide the terminology and notation for this paper.

\section{INTERVAL SETS}

Let $U$ be a set and let $X, Y$ be subsets of $U$. The functor $[X, Y]_{\mathrm{I}}$ yielding a family of subsets of $U$ is defined by:

(Def. 1) $[X, Y]_{\mathrm{I}}=\{A \subseteq U: X \subseteq A \wedge A \subseteq Y\}$.

In the sequel $U$ denotes a set and $X, Y$ denote subsets of $U$.

Next we state several propositions:

(1) For every set $x$ holds $x \in[X, Y]_{\mathrm{I}}$ iff $X \subseteq x \subseteq Y$.

(2) If $[X, Y]_{\mathrm{I}} \neq \emptyset$, then $X, Y \in[X, Y]_{\mathrm{I}}$. 
(3) For every set $U$ and for all subsets $A, B$ of $U$ such that $A \nsubseteq B$ holds $[A, B]_{\mathrm{I}}=\emptyset$.

(4) For every set $U$ and for all subsets $A, B$ of $U$ such that $[A, B]_{\mathrm{I}}=\emptyset$ holds $A \nsubseteq B$.

(5) For all subsets $A, B$ of $U$ such that $[A, B]_{\mathrm{I}} \neq \emptyset$ holds $A \subseteq B$.

(6) For all subsets $A, B, C, D$ of $U$ such that $[A, B]_{\mathrm{I}} \neq \emptyset$ and $[A, B]_{\mathrm{I}}=$ $[C, D]_{\mathrm{I}}$ holds $A=C$ and $B=D$.

(7) For every non empty set $U$ and for every non empty subset $A$ of $U$ holds $\left[A, \emptyset_{U}\right]_{\mathrm{I}}=\emptyset$.

(8) For every subset $A$ of $U$ holds $[A, A]_{\mathrm{I}}=\{A\}$.

Let us consider $U$. A family of subsets of $U$ is said to be an interval set of $U$ if:

(Def. 2) There exist subsets $A, B$ of $U$ such that it $=[A, B]_{\mathrm{I}}$.

We now state two propositions:

(9) For every non empty set $U$ holds $\emptyset$ is an interval set of $U$.

(10) For every non empty set $U$ and for every subset $A$ of $U$ holds $\{A\}$ is an interval set of $U$.

Let us consider $U$ and let $A, B$ be subsets of $U$. Then $[A, B]_{\mathrm{I}}$ is an interval set of $U$.

Let $U$ be a non empty set. Note that there exists an interval set of $U$ which is non empty.

We now state three propositions:

(11) Let $U$ be a non empty set and $A$ be a set. Then $A$ is a non empty interval set of $U$ if and only if there exist subsets $A_{1}, A_{2}$ of $U$ such that $A_{1} \subseteq A_{2}$ and $A=\left[A_{1}, A_{2}\right]_{\mathrm{I}}$.

(12) Let $U$ be a non empty set and $A_{1}, A_{2}, B_{1}, B_{2}$ be subsets of $U$. If $A_{1} \subseteq A_{2}$ and $B_{1} \subseteq B_{2}$, then $\left[A_{1}, A_{2}\right]_{\mathrm{I}} \cap\left[B_{1}, B_{2}\right]_{\mathrm{I}}=\{C ; C$ ranges over subsets of $U$ : $\left.A_{1} \cap B_{1} \subseteq C \wedge C \subseteq A_{2} \cap B_{2}\right\}$.

(13) Let $U$ be a non empty set and $A_{1}, A_{2}, B_{1}, B_{2}$ be subsets of $U$. If $A_{1} \subseteq A_{2}$ and $B_{1} \subseteq B_{2}$, then $\left[A_{1}, A_{2}\right]_{\mathrm{I}} \uplus\left[B_{1}, B_{2}\right]_{\mathrm{I}}=\{C ; C$ ranges over subsets of $U$ : $\left.A_{1} \cup B_{1} \subseteq C \wedge C \subseteq A_{2} \cup B_{2}\right\}$.

Let $U$ be a non empty set and let $A, B$ be non empty interval sets of $U$. The functor $A \cap_{\mathrm{I}} B$ yielding an interval set of $U$ is defined by:

(Def. 3) $\quad A \cap_{\mathrm{I}} B=A \cap B$.

The functor $A \cup_{\mathrm{I}} B$ yields an interval set of $U$ and is defined by:

(Def. 4) $\quad A \cup_{\mathrm{I}} B=A \cup B$.

Let $U$ be a non empty set and let $A, B$ be non empty interval sets of $U$. Note that $A \cap_{\mathrm{I}} B$ is non empty and $A \cup_{\mathrm{I}} B$ is non empty. 
In the sequel $U$ denotes a non empty set and $A, B, C$ denote non empty interval sets of $U$.

Let us consider $U, A$. The functor $A_{1}$ yielding a subset of $U$ is defined by: (Def. 5) There exists a subset $B$ of $U$ such that $A=\left[A_{1}, B\right]_{\mathrm{I}}$.

The functor $A_{2}$ yielding a subset of $U$ is defined as follows:

(Def. 6) There exists a subset $B$ of $U$ such that $A=\left[B, A_{\mathbf{2}}\right]_{\mathrm{I}}$.

We now state several propositions:

(14) For every set $X$ holds $X \in A$ iff $A_{\mathbf{1}} \subseteq X \subseteq A_{\mathbf{2}}$.

(15) $A=\left[A_{1}, A_{2}\right]_{\mathrm{I}}$.

(16) $A_{1} \subseteq A_{2}$.

(17) $A \cup_{\mathrm{I}} B=\left[A_{\mathbf{1}} \cup B_{\mathbf{1}}, A_{\mathbf{2}} \cup B_{\mathbf{2}}\right]_{\mathrm{I}}$.

(18) $A \cap_{\mathrm{I}} B=\left[A_{\mathbf{1}} \cap B_{\mathbf{1}}, A_{\mathbf{2}} \cap B_{\mathbf{2}}\right]_{\mathrm{I}}$.

Let us consider $U$ and let us consider $A, B$. Let us observe that $A=B$ if and only if:

(Def. 7) $A_{1}=B_{1}$ and $A_{2}=B_{2}$.

The following propositions are true:

(19) $A \cup_{\mathrm{I}} A=A$.

(20) $A \cap_{\mathrm{I}} A=A$.

(21) $A \cup_{\mathrm{I}} B=B \cup_{\mathrm{I}} A$.

(22) $A \cap_{\mathrm{I}} B=B \cap_{\mathrm{I}} A$.

(23) $\left(A \cup_{\mathrm{I}} B\right) \cup_{\mathrm{I}} C=A \cup_{\mathrm{I}}\left(B \cup_{\mathrm{I}} C\right)$.

(24) $\left(A \cap_{\mathrm{I}} B\right) \cap_{\mathrm{I}} C=A \cap_{\mathrm{I}}\left(B \cap_{\mathrm{I}} C\right)$.

Let $X$ be a set and let $F$ be a family of subsets of $X$. We say that $F$ is ordered if and only if:

(Def. 8) There exist sets $A, B$ such that $A, B \in F$ and for every set $Y$ holds $Y \in F$ iff $A \subseteq Y \subseteq B$.

Let $X$ be a set. Observe that there exists a family of subsets of $X$ which is non empty and ordered.

Next we state two propositions:

(25) For all subsets $A, B$ of $U$ such that $A \subseteq B$ holds $[A, B]_{\mathrm{I}}$ is a non empty ordered family of subsets of $U$.

(26) Every non empty interval set of $U$ is a non empty ordered family of subsets of $U$.

Let $X$ be a set. We introduce $\min X$ as a synonym of $\bigcap X$. We introduce $\max X$ as a synonym of $\bigcup X$.

Let $X$ be a set and let $F$ be a non empty ordered family of subsets of $X$. Then $\min F$ is an element of $F$. Then $\max F$ is an element of $F$.

We now state a number of propositions: 
(27) Let $A, B$ be subsets of $U$ and $F$ be an ordered non empty family of subsets of $U$. If $F=[A, B]_{\mathrm{I}}$, then $\min F=A$ and $\max F=B$.

(28) For all sets $X, Y$ and for every non empty ordered family $A$ of subsets of $X$ holds $Y \in A$ iff $\min A \subseteq Y \subseteq \max A$.

(29) For every set $X$ and for all non empty ordered families $A, B, C$ of subsets of $X$ holds $A \uplus B \cap C=(A \uplus B) \cap(A \uplus C)$.

(30) For every set $X$ and for all non empty ordered families $A, B, C$ of subsets of $X$ holds $A$ ก $(B \uplus C)=A \cap B \uplus A \cap C$.

(31) $A \cup_{\mathrm{I}} B \cap_{\mathrm{I}} C=\left(A \cup_{\mathrm{I}} B\right) \cap_{\mathrm{I}}\left(A \cup_{\mathrm{I}} C\right)$.

(32) $A \cap_{\mathrm{I}}\left(B \cup_{\mathrm{I}} C\right)=A \cap_{\mathrm{I}} B \cup_{\mathrm{I}} A \cap_{\mathrm{I}} C$.

(33) For every set $X$ and for all non empty ordered families $A, B$ of subsets of $X$ holds $A$ ก $(A \uplus B)=A$.

(34) For every set $X$ and for all non empty ordered families $A, B$ of subsets of $X$ holds $A \cap B \uplus B=B$.

(35) $A \cap_{\mathrm{I}}\left(A \cup_{\mathrm{I}} B\right)=A$.

(36) $A \cap_{\mathrm{I}} B \cup_{\mathrm{I}} B=B$.

\section{Families of Subsets}

One can prove the following propositions:

(37) For every non empty set $U$ and for all families $A, B$ of subsets of $U$ holds $A \backslash \backslash B$ is a family of subsets of $U$.

(38) Let $U$ be a non empty set and $A, B$ be non empty ordered families of subsets of $U$. Then $A \backslash \backslash B=\{C \subseteq U: \min A \backslash \max B \subseteq C \wedge C \subseteq$ $\max A \backslash \min B\}$.

(39) Let $U$ be a non empty set and $A_{1}, A_{2}, B_{1}, B_{2}$ be subsets of $U$. If $A_{1} \subseteq A_{2}$ and $B_{1} \subseteq B_{2}$, then $\left[A_{1}, A_{2}\right]_{\mathrm{I}} \backslash \backslash\left[B_{1}, B_{2}\right]_{\mathrm{I}}=\left\{C \subseteq U: A_{1} \backslash B_{2} \subseteq C \wedge C \subseteq\right.$ $\left.A_{2} \backslash B_{1}\right\}$.

Let $U$ be a non empty set and let $A, B$ be non empty interval sets of $U$. The functor $A \backslash_{\mathrm{I}} B$ yields an interval set of $U$ and is defined as follows:

(Def. 9) $A \backslash_{\mathrm{I}} B=A \backslash \backslash B$.

Let $U$ be a non empty set and let $A, B$ be non empty interval sets of $U$. Observe that $A \backslash_{\mathrm{I}} B$ is non empty.

Next we state several propositions:

(40) $A \backslash_{\mathrm{I}} B=\left[A_{\mathbf{1}} \backslash B_{\mathbf{2}}, A_{\mathbf{2}} \backslash B_{\mathbf{1}}\right]_{\mathrm{I}}$.

(41) For all subsets $X, Y$ of $U$ such that $A=[X, Y]_{\mathrm{I}}$ holds $A \backslash_{\mathrm{I}} C=$ $\left[X \backslash C_{\mathbf{2}}, Y \backslash C_{\mathbf{1}}\right]_{\mathrm{I}}$.

(42) For all subsets $X, Y, W, Z$ of $U$ such that $A=[X, Y]_{\mathrm{I}}$ and $C=[W, Z]_{\mathrm{I}}$ holds $A \backslash_{\mathrm{I}} C=[X \backslash Z, Y \backslash W]_{\mathrm{I}}$. 
(43) For every non empty set $U$ holds $\left[\Omega_{U}, \Omega_{U}\right]_{\mathrm{I}}$ is a non empty interval set of $U$.

(44) For every non empty set $U$ holds $\left[\emptyset_{U}, \emptyset_{U}\right]_{\mathrm{I}}$ is a non empty interval set of $U$.

Let $U$ be a non empty set. Note that $\left[\Omega_{U}, \Omega_{U}\right]_{\mathrm{I}}$ is non empty and $\left[\emptyset_{U}, \emptyset_{U}\right]_{\mathrm{I}}$ is non empty.

Let $U$ be a non empty set and let $A$ be a non empty interval set of $U$. The functor $-A$ yielding a non empty interval set of $U$ is defined as follows:

(Def. 10) $-A=\left[\Omega_{U}, \Omega_{U}\right]_{\mathrm{I}} \backslash_{\mathrm{I}} A$.

We now state four propositions:

(45) For every non empty set $U$ and for every non empty interval set $A$ of $U$ holds $-A=\left[\left(A_{\mathbf{2}}\right)^{\mathrm{c}},\left(A_{\mathbf{1}}\right)^{\mathrm{c}}\right]_{\mathrm{I}}$.

(46) For all subsets $X, Y$ of $U$ such that $A=[X, Y]_{\mathrm{I}}$ and $X \subseteq Y$ holds $-A=\left[Y^{\mathrm{c}}, X^{\mathrm{c}}\right]_{\mathrm{I}}$.

(47) $-\left[\emptyset_{U}, \emptyset_{U}\right]_{\mathrm{I}}=\left[\Omega_{U}, \Omega_{U}\right]_{\mathrm{I}}$.

(48) $-\left[\Omega_{U}, \Omega_{U}\right]_{\mathrm{I}}=\left[\emptyset_{U}, \emptyset_{U}\right]_{\mathrm{I}}$.

\section{Counterexamples}

Next we state several propositions:

(49) There exists a non empty interval set $A$ of $U$ such that $A \cap_{\mathrm{I}}-A \neq$ $\left[\emptyset_{U}, \emptyset_{U}\right]_{\mathrm{I}}$

(50) There exists a non empty interval set $A$ of $U$ such that $A \cup_{\mathrm{I}}-A \neq$ $\left[\Omega_{U}, \Omega_{U}\right]_{\mathrm{I}}$.

(51) There exists a non empty interval set $A$ of $U$ such that $A \backslash_{\mathrm{I}} A \neq\left[\emptyset_{U}, \emptyset_{U}\right]_{\mathrm{I}}$.

(52) For every non empty interval set $A$ of $U$ holds $U \in A \cup_{\mathrm{I}}-A$.

(53) For every non empty interval set $A$ of $U$ holds $\emptyset \in A \cap_{\mathrm{I}}-A$.

(54) For every non empty interval set $A$ of $U$ holds $\emptyset \in A \backslash_{\mathrm{I}} A$.

\section{Lattice of Interval Sets}

Let $U$ be a non empty set. The functor $\mathrm{I}\left(2^{U}\right)$ yielding a non empty set is defined by:

(Def. 11) For every set $x$ holds $x \in \mathrm{I}\left(2^{U}\right)$ iff $x$ is a non empty interval set of $U$.

Let $U$ be a non empty set. The functor InterLatt $U$ yields a strict non empty lattice structure and is defined by the conditions (Def. 12).

(Def. 12)(i) The carrier of InterLatt $U=\mathrm{I}\left(2^{U}\right)$, and

(ii) for all elements $a, b$ of the carrier of InterLatt $U$ and for all non empty interval sets $a^{\prime}, b^{\prime}$ of $U$ such that $a^{\prime}=a$ and $b^{\prime}=b$ holds (the join operation 
of InterLatt $U)(a, b)=a^{\prime} \cup_{\mathrm{I}} b^{\prime}$ and (the meet operation of InterLatt $\left.U\right)(a$, b) $=a^{\prime} \cap_{\mathrm{I}} b^{\prime}$.

Let $U$ be a non empty set. Observe that InterLatt $U$ is lattice-like.

Let $X$ be a tolerance space.

(Def. 13) An element of $2^{\text {the carrier of } X} \times 2^{\text {the carrier of } X}$ is said to be a rough set of $X$.

One can prove the following proposition

(55) For every tolerance space $X$ and for every rough set $A$ of $X$ there exist subsets $B, C$ of $X$ such that $A=\langle B, C\rangle$.

Let $X$ be a tolerance space and let $A$ be a subset of $X$. The functor RS $A$ yielding a rough set of $X$ is defined by:

(Def. 14) $\operatorname{RS} A=\langle\operatorname{LAp}(A), \operatorname{UAp}(A)\rangle$.

Let $X$ be a tolerance space and let $A$ be a rough set of $X$. The functor $\mathrm{LAp}(A)$ yielding a subset of $X$ is defined as follows:

(Def. 15) $\operatorname{LAp}(A)=A_{1}$.

The functor $\operatorname{UAp}(A)$ yielding a subset of $X$ is defined by:

(Def. 16) $\operatorname{UAp}(A)=A_{\mathbf{2}}$.

Let $X$ be a tolerance space and let $A, B$ be rough sets of $X$. Let us observe that $A=B$ if and only if:

(Def. 17) $\operatorname{LAp}(A)=\operatorname{LAp}(B)$ and $\operatorname{UAp}(A)=\operatorname{UAp}(B)$.

Let $X$ be a tolerance space and let $A, B$ be rough sets of $X$. The functor $A \cup_{\mathrm{I}} B$ yields a rough set of $X$ and is defined by:

(Def. 18) $\quad A \cup_{\mathrm{I}} B=\langle\operatorname{LAp}(A) \cup \operatorname{LAp}(B), \operatorname{UAp}(A) \cup \operatorname{UAp}(B)\rangle$.

The functor $A \cap_{\mathrm{I}} B$ yielding a rough set of $X$ is defined as follows:

(Def. 19) $A \cap_{\mathrm{I}} B=\langle\operatorname{LAp}(A) \cap \operatorname{LAp}(B), \operatorname{UAp}(A) \cap \operatorname{UAp}(B)\rangle$.

In the sequel $X$ denotes a tolerance space and $A, B, C$ denote rough sets of $X$.

Next we state a number of propositions:

(56) $\operatorname{LAp}(A \cup \mathrm{I} B)=\operatorname{LAp}(A) \cup \operatorname{LAp}(B)$.

(57) $\operatorname{UAp}(A \cup B)=\operatorname{UAp}(A) \cup \operatorname{UAp}(B)$.

(58) $\operatorname{LAp}\left(A \cap_{\mathrm{I}} B\right)=\operatorname{LAp}(A) \cap \operatorname{LAp}(B)$.

(59) $\operatorname{UAp}\left(A \cap_{\mathrm{I}} B\right)=\operatorname{UAp}(A) \cap \operatorname{UAp}(B)$.

(60) $A \cup_{\mathrm{I}} A=A$.

(61) $A \cap_{\mathrm{I}} A=A$.

(62) $A \cup_{\mathrm{I}} B=B \cup_{\mathrm{I}} A$.

(63) $A \cap_{\mathrm{I}} B=B \cap_{\mathrm{I}} A$.

(64) $\left(A \cup_{\mathrm{I}} B\right) \cup_{\mathrm{I}} C=A \cup_{\mathrm{I}}\left(B \cup_{\mathrm{I}} C\right)$.

(65) $\left(A \cap_{\mathrm{I}} B\right) \cap_{\mathrm{I}} C=A \cap_{\mathrm{I}}\left(B \cap_{\mathrm{I}} C\right)$. 
(66) $A \cap_{\mathrm{I}}\left(B \cup_{\mathrm{I}} C\right)=A \cap_{\mathrm{I}} B \cup_{\mathrm{I}} A \cap_{\mathrm{I}} C$.

(67) $A \cup_{\mathrm{I}} A \cap_{\mathrm{I}} B=A$.

(68) $A \cap_{\mathrm{I}}\left(A \cup_{\mathrm{I}} B\right)=A$.

\section{Lattice of Rough Sets}

Let us consider $X$. The functor RoughSets $X$ is defined as follows:

(Def. 20) For every set $x$ holds $x \in \operatorname{RoughSets} X$ iff $x$ is a rough set of $X$.

Let us consider $X$. One can check that RoughSets $X$ is non empty.

Let us consider $X$ and let $R$ be an element of RoughSets $X$. The functor ${ }^{\circledR} R$ yielding a rough set of $X$ is defined by:

(Def. 21) ${ }^{\circledR} R=R$.

Let us consider $X$ and let $R$ be a rough set of $X$. The functor ${ }^{\circledR} R$ yielding an element of RoughSets $X$ is defined as follows:

(Def. 22) ${ }^{\circledR} R=R$.

Let us consider $X$. The functor RSLattice $X$ yields a strict lattice structure and is defined by the conditions (Def. 23).

(Def. 23)(i) The carrier of RSLattice $X=\operatorname{RoughSets} X$, and

(ii) for all elements $A, B$ of RoughSets $X$ and for all rough sets $A^{\prime}, B^{\prime}$ of $X$ such that $A=A^{\prime}$ and $B=B^{\prime}$ holds (the join operation of RSLattice $\left.X\right)(A$, $B)=A^{\prime} \cup_{\mathrm{I}} B^{\prime}$ and (the meet operation of RSLattice $\left.X\right)(A, B)=A^{\prime} \cap_{\mathrm{I}} B^{\prime}$.

Let us consider $X$. Observe that RSLattice $X$ is non empty.

Let us consider $X$. Observe that RSLattice $X$ is lattice-like.

Let us consider $X$. Note that RSLattice $X$ is distributive. by:

Let us consider $X$. The functor ERS $X$ yields a rough set of $X$ and is defined

(Def. 24) $\quad$ ERS $X=\langle\emptyset, \emptyset\rangle$.

One can prove the following proposition

(69) For every rough set $A$ of $X$ holds ERS $X \cup_{\mathrm{I}} A=A$.

Let us consider $X$. The functor $\operatorname{TRS}(X)$ is a rough set of $X$ and is defined as follows:

(Def. 25) $\operatorname{TRS}(X)=\left\langle\Omega_{X}, \Omega_{X}\right\rangle$.

One can prove the following proposition

(70) For every rough set $A$ of $X$ holds $\operatorname{TRS}(X) \cap_{\mathrm{I}} A=A$.

Let us consider $X$. Note that RSLattice $X$ is bounded.

We now state the proposition

(71) Let $X$ be a tolerance space, $A, B$ be elements of RSLattice $X$, and $A^{\prime}$, $B^{\prime}$ be rough sets of $X$. If $A=A^{\prime}$ and $B=B^{\prime}$, then $A \sqsubseteq B$ iff $\operatorname{LAp}\left(A^{\prime}\right) \subseteq$ $\operatorname{LAp}\left(B^{\prime}\right)$ and $\operatorname{UAp}\left(A^{\prime}\right) \subseteq \operatorname{UAp}\left(B^{\prime}\right)$. 
Let us consider $X$. Observe that RSLattice $X$ is complete.

\section{REFERENCES}

[1] Grzegorz Bancerek. Complete lattices. Formalized Mathematics, 2(5):719-725, 1991.

[2] Czesław Byliński. Binary operations. Formalized Mathematics, 1(1):175-180, 1990.

[3] Adam Grabowski. Basic properties of rough sets and rough membership function. Formalized Mathematics, 12(1):21-28, 2004.

[4] Amin Mousavi and Parviz Jabedar-Maralani. Relative sets and rough sets. Int. J. Appl. Math. Comput. Sci., 11(3):637-653, 2001.

[5] Beata Padlewska. Families of sets. Formalized Mathematics, 1(1):147-152, 1990.

[6] Z. Pawlak. Rough sets. International Journal of Parallel Programming, 11:341-356, 1982, doi:10.1007/BF01001956.

[7] Andrzej Trybulec. Tuples, projections and Cartesian products. Formalized Mathematics, 1(1):97-105, 1990.

[8] Zinaida Trybulec. Properties of subsets. Formalized Mathematics, 1(1):67-71, 1990.

[9] Y.Y. Yao. Interval-set algebra for qualitative knowledge representation. Proc. 5-th Int. Conf. Computing and Information, pages 370-375, 1993.

[10] Stanisław Żukowski. Introduction to lattice theory. Formalized Mathematics, 1(1):215$222,1990$.

Received October 10, 2009 